\title{
AVOIDING HARD DECISIONS IN ADAPTIVE WATERMARKING
}

\author{
M. Schlauweg, D. Pröfrock, and E. Müller \\ Institute of Communications Engineering, \\ Faculty of Computer Science and Electrical Engineering, University of Rostock, \\ Rostock 18119, Germany
}

\begin{abstract}
In this paper, we describe the coupling of content adaptive watermark decoding and soft-decision forward error correction. We deduce three fundamental properties from adaptive watermarking methods switching between different embedding strengths. It is shown how a weighting process at the decoder can be used to build up an overall soft processing watermarking. No pre-distortion has to be used, and hence, the image quality is not degraded. In this conjunction, even adaptation techniques can be used where it is computational infeasible to project a pre-distortion back onto the host image. Experimental results indicate the improvement in bit error correction during watermark retrieval by a factor of 5 . Without loss of security, performance or functionality our new technique can be integrated very easily into numerous watermarking applications.
\end{abstract}

Index Terms - Digital watermarking, image segmentation, adaptive decoding, soft-decision forward error correction

\section{INTRODUCTION}

In recent years, several image watermarking methods have been proposed that adjust the embedding strength depending on the host signal [1]. More sophisticated approaches employ models of the human visual system to keep embedding induced distortions below a just noticeable difference threshold [2]. They exploit the sensitivity of human's eye to luminance masking, frequency masking as well as contrast masking. Parameters determined from the host signal (e.g., applying Watson's perceptual metric) are used for weighting the watermark. Other methods take advantage of the property that the human visual system is less sensitive to changes in textured regions than in smooth regions of an image. After a texture region separation, data can be embedded with higher robustness against watermark attacks in stronger textured regions [3].

However, in content adaptive watermarking approaches during the extraction process the embedding strength parameters or the region separation map have to be known. If the system is blind, that means, if the used parameters are not transmitted besides the watermarked image and/or if the original image is unknown to the extractor, these parameters have to be determined from the watermarked image. Slight changes due to attacks can yield errors. Even if no attack has been applied discrepancies can occur [4], for example, because the embedding process itself has influence on the parameter calculation and the separation feature map.

In the case of region separation-based adaptation the most often applied technique to circumvent discrepancies between both separation feature maps is to form a gap around the separation threshold. In other words, the used feature is pre-distorted to leave a margin. As a consequence the image quality is degraded. Furthermore, there are separation approaches where it is computational infeasible or even impossible to project the pre-distortion back onto the host image. For example, the separation feature could have been calculated using higher order statistics from extensive data. In this case, errors are ignored or additionally have to be corrected by FEC (forward error correction).

In this paper, we present a new solution to this problem. We integrate the commonly used hard region separation into an overall soft processing framework, as in Fig. 1 c). The FEC-decoder input signal is weighted using the knowledge of how close the feature is to the separation threshold.

a)

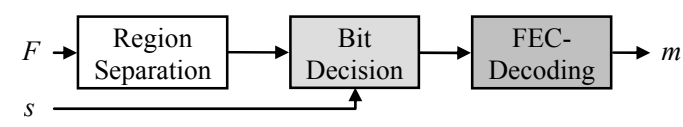

b)

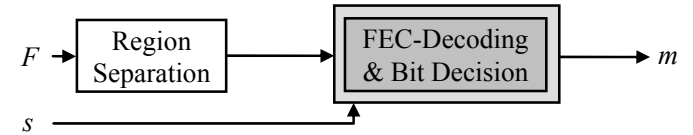

c)

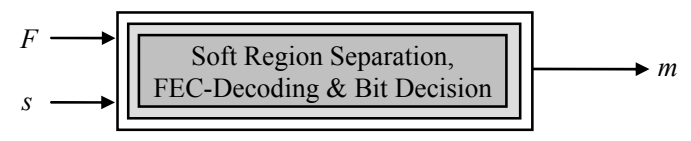

Fig. 1. Overall hard processing a), hard region separation with soft bit decoding b), overall soft processing c). $F$ is the separation feature, $s$ is the host signal, $m$ is the watermark.

\section{SOFT-DECODED ADAPTIVE WATERMARKING}

If there are discrepancies of the region separation during adaptively embedding and extracting the watermark $m$, as in 


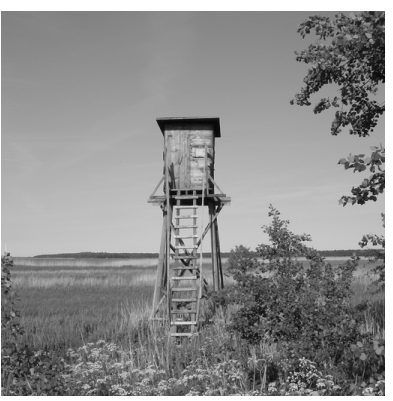

a)

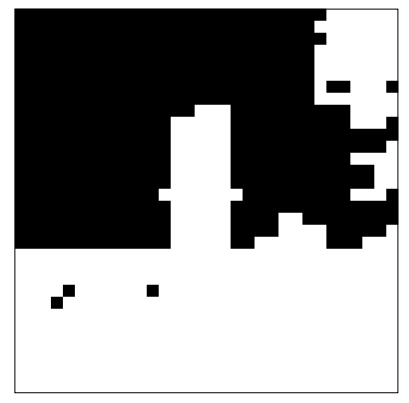

b)

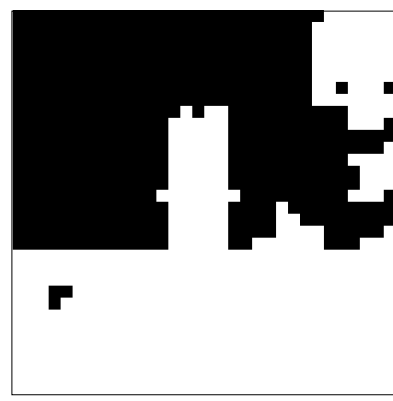

c)

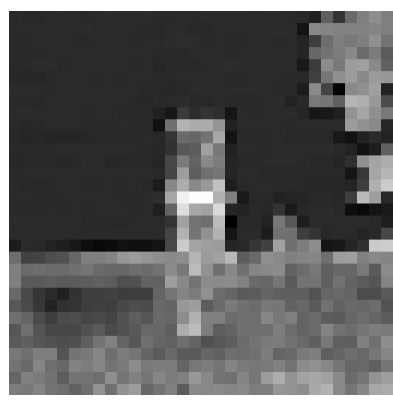

d)

Fig. 2. Original image a). Hard embedding mask b), hard extraction mask after lossy JPEG compression with quality factor $\mathrm{QF}=50 \mathrm{c}$ ), and soft extraction mask d) using the new soft texture segmentation $(\tau=2)$.

Fig. 2 b) and c), wrong parameters are used and bit errors can occur. For example, suppose that during the embedding process the watermarking strength $\Delta_{1}$ has been used for all black blocks and $\Delta_{2}$ has been used for the white blocks. Now, at the decoder, the nine mask differences in Fig. 2 c) would result in falsely used extraction strengths. Thus, the first goal must be to find a segmentation scheme that yields less decision errors. Furthermore, the separation feature map $F$ must consist of real values, $F \in \mathbb{R}$. These soft values, the soft mask, can be used during soft-decision decoding for weighting the input signal $s$.

\subsection{DWT-based soft texture mask generation}

In [3], we applied texture segmentation in the DWT domain (discrete wavelet transform) for an image authentication scheme. Large DWT coefficients indicate image positions with strong texture, whereas small ones stand for homogenous regions. Except for the $\mathrm{LL}^{4}$-subband, all coefficients of the fourth decomposition level were compared to a threshold. Afterwards, the known morphologic operations closing and erosion were used to eliminate small gaps and to refine the separation. The binary masks were combined logically and used during embedding as well as extraction to select between two different watermarking strengths $\Delta_{1}$ and $\Delta_{2}$.

But the described DWT segmentation approach was originally designed for a hard region separation (with predistortion) and hard FEC-decoding. Now, in this paper we modify it to a soft version, whereas the texture threshold $\tau$ is subtracted rather than being used for binarization. The soft feature map is shown for a common photo in Fig. 2 d). The homogenous sky yields negative values, whereas for stronger textured regions the feature is positive.

\subsection{Three key properties towards soft decoding}

For watermark embedding we use QIM (quantization index modulation) in the DWT domain as described in [3]. But for the extraction site, we found out three interesting properties to be used for soft-decoding during nearest-neighbor quan- tization. Thereby, $\Lambda_{1}$ and $\Lambda_{2}$ denote the two quantization lattices that each consist of sub-lattices marked with $\mathbf{x}$ and $\mathbf{0}$ accordingly. Although here we describe the embedding for the DWT domain, other domains, e.g., DCT (discrete cosine transform) or pixel domain could be used as well.

\subsubsection{Property I - Lattice point coverage}

In Fig. 3, the "natural" covers of $\Lambda_{1}$ and $\Lambda_{2}$ are shown for the case of lattice crossings due to false feature separation. The shaded areas indicate "natural positive crossings". For example, suppose $\Lambda_{1}$ has been used during embedding and the host signal was quantized to the highlighted point $\mathbf{0}$. If afterwards the mask changes slightly as in Fig. 2 c), then $\Lambda_{2}$ would be used during extraction for this sample. In this case, the nearest-neighbor quantization would yield the correct bit decision as long as the sample keeps inside the shaded area, even if the separation feature has passed over the decision threshold. But if the point $\mathbf{x}$ right beside it has been used, it would be falsely decided to a point 0 in lattice $\Lambda_{2}$. In this case, a bit substitution error would occur.

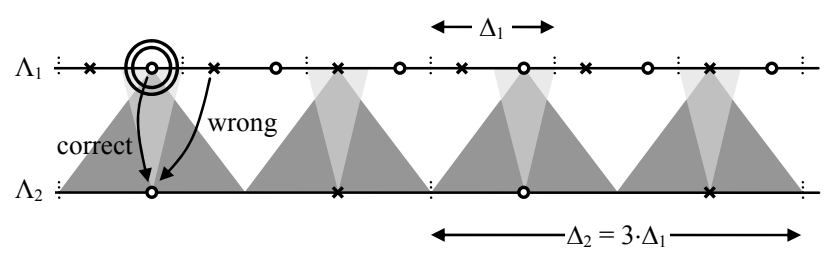

Fig. 3. Covers of two QIM lattices $\left(\Delta_{2}=3 \cdot \Delta_{1}\right)$.

However, the possibility is highest that no error occurs during a transition $\Lambda_{1} \leftrightarrow \Lambda_{2}$ in the case of an exactly three times larger quantization interval $\Delta_{2}$. Thereby, in at least 50 percent of all cases there is no problem if a transition occurs if the distortion to the quantized signal is less than $\Delta_{1} / 2$.

\subsubsection{Property II - Distortion Probability Density Function} In [5], Vila-Forcén et al studied additive attacks against quantization-based watermarking. For attacks, such as lossy compression, noise adding or filtering, the distortion to the 
quantized signal can be expected to be Gaussian distributed. Since the variance of this distribution is the same for both lattices $\Lambda_{1}$ and $\Lambda_{2}$, the following distortion probability density functions $p d f\left(s_{\mathrm{i}}\right)$ can be expected (see Fig. 4). Due to the quantization $s_{\mathrm{i}}=\left\{-1 \leq s_{\mathrm{i}} \leq+1: s_{\mathrm{i}} \in \mathbb{R}\right\}, \forall \mathrm{i} \in\{1,2\}$ for one periodical quantization bin, whereas $s_{1}=s / \Delta_{1}$ and $s_{2}=s / \Delta_{2}$.
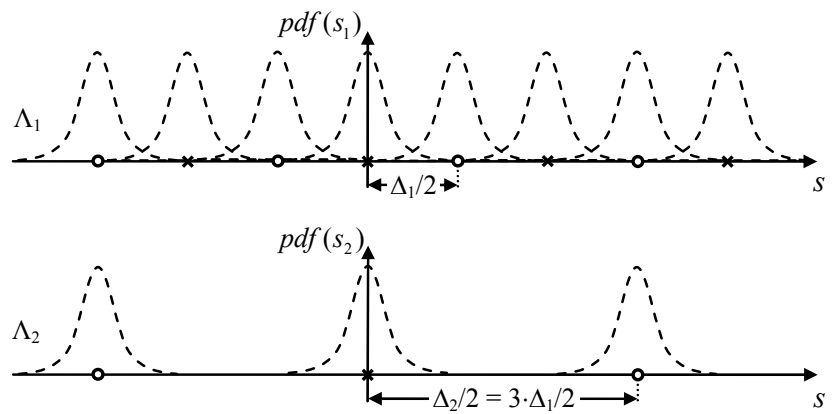

Fig. 4. Probability density functions of the disturbed signal.

Both probability density functions are drawn one upon the other to better visualize that there are spaces at the second lattice where it is unlikely that a signal sample is located. In other words, if in this case the feature is close to the decision threshold and the signal sample is somewhere in the space where $\operatorname{pdf}\left(s_{\mathrm{i}}\right)$ is small, it is more likely that the sample was originally embedded using lattice $\Lambda_{1}$.

\subsubsection{Property III - Certainty of Decision}

We define $C$ as certainty of how close the input feature $F$ is to the selected feature threshold $\tau$. That means if the feature is close to the decision threshold, it is uncertain which QIM lattice has to be used. In this case the certainty is zero. If the feature is far from the threshold and it is oblivious which lattice was chosen during embedding, the certainty is high. Using this certainty we propose two weighting functions, $f_{1}$ and $f_{2}$, for the input signals in $\Lambda_{1}$ and $\Lambda_{2}$. Thereby $s_{1}$ and $s_{2}$ will be coupled to the soft key properties described above.

$$
\begin{aligned}
& C=F-\tau \\
& f_{1}(C)=\frac{1}{2}-\frac{\arctan (C)}{\pi} \\
& f_{2}\left(C, s_{2}, O\right)=1-f_{1}\left(C \cdot\left(\left|s_{2}\right|+O\right)\right) \\
& \begin{array}{c|c|c}
C_{\mathrm{x}} & f_{1}(C) & f_{2}\left(C, s_{2}, O\right) \\
\hline 0 & 0,5 & 0,5 \\
\hline-\infty & 1 & 0 \\
\hline+\infty & 0 & 1
\end{array}
\end{aligned}
$$

The weighting function $f_{2}$ for lattice $\Lambda_{2}$ depends on the absolute value of input signal $s_{2}$ to implement Property II. The value $O$ should be set to the ratio $\Delta_{1} / \Delta_{2}$, e.g., $O=1 / 3$. For one periodical quantization bin the weighting functions are visualized in Fig. 5. Considering the input signal both functions are opposed to each other.
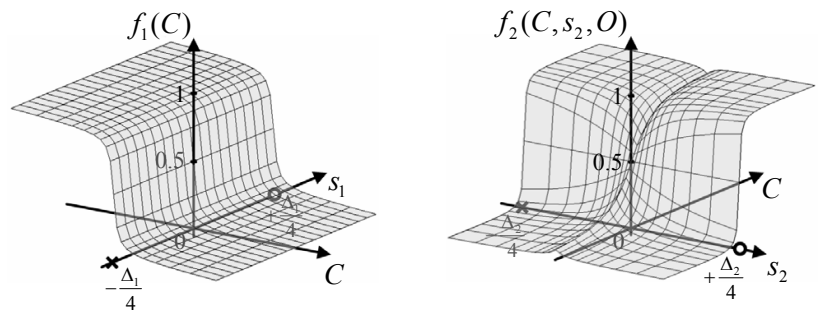

Fig. 5. Soft feature input signal weighting functions.

Using these weighting functions the input signal can be coupled to the feature decision in a soft processing manner.

$$
s_{\mathrm{w}}=\frac{s_{1} \cdot f_{1}(C)+s_{2} \cdot f_{2}\left(C, s_{2}, O\right)}{2}
$$

It is clear that data embedded using the weaker embedding strength $\Delta_{1}$ is less robust to attacks or common signal processing than data embedded using $\Delta_{2}$ (see Fig. 6). Hence, we stretch the signal $s_{\mathrm{W}}$ by the factor $\Delta_{2} / \Delta_{1}$ at all mask positions where the separation feature is larger than the threshold $\tau$. This weighting results in a stronger influence of the information embedded using $\Lambda_{2}$ during FEC-decoding.

$$
s_{\mathrm{w}}^{*}=\left\{\begin{array}{cc}
s_{\mathrm{w}} & C<0 \\
\Delta_{2} / \Delta_{1} \cdot s_{\mathrm{w}} & C \geq 0
\end{array}\right.
$$

\subsection{Soft-decision decoding of the weighted signal}

After the weighing process, the FEC-decoder receives a stream containing information about the reliability of each received symbol. For example, the popular Viterbi-Decoder with soft-decision input used to decode convolutional codes can be employed to determine the embedded message $m$. By observing a sequence of symbols the decoder determines multiple paths of message states in a kind of state machine. Each of these states is valuated by a path metric, representing the reliability of each bit-decision. Afterwards, in a trace back process the maximum likelihood path survives.

Our input signal weighting approach directly influences the decoder metric without the need for reprogramming the source code of the used FEC-decoder. Hence, this new preweighting results in two advantages. First, the implementation effort is very low, and second, any soft-input FECdecoder can be used as well as simply exchanged.

\section{EXPERIMENTAL RESULTS}

The probability of mask transitions using the described DWT-based soft region segmentation is very low as can be 
seen in Fig. 6. Thus, we had to perform a huge number of simulations to assess the improvement in bit error correction during watermark retrieval. Therefore, in multiple runs we embedded 1024 bit of random data into each of a set of 32 natural photos (512×512 pixels).
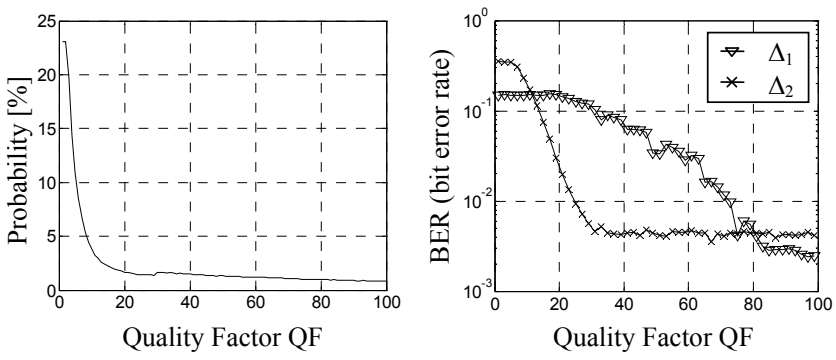

Fig. 6. Probability of occurrence of mask transitions (left) and extraction bit error rate depending on the used embedding strength (right) after JPEG compression with QF.

To get an impression of how the image from Fig. 2 a) is changed due to the embedding process, in Fig. 7 the watermarked image is displayed.

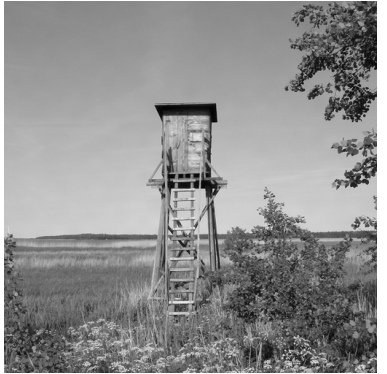

a)

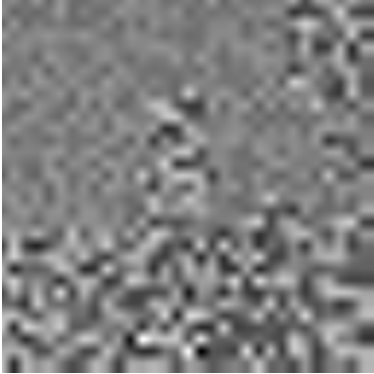

b)
Fig. 7. Adaptively watermarked image a), $\Delta_{2}=3 \cdot \Delta_{1}$, PSNR $=45.91 \mathrm{~dB}$. Difference to original $\mathrm{b}$ ) (scaled).

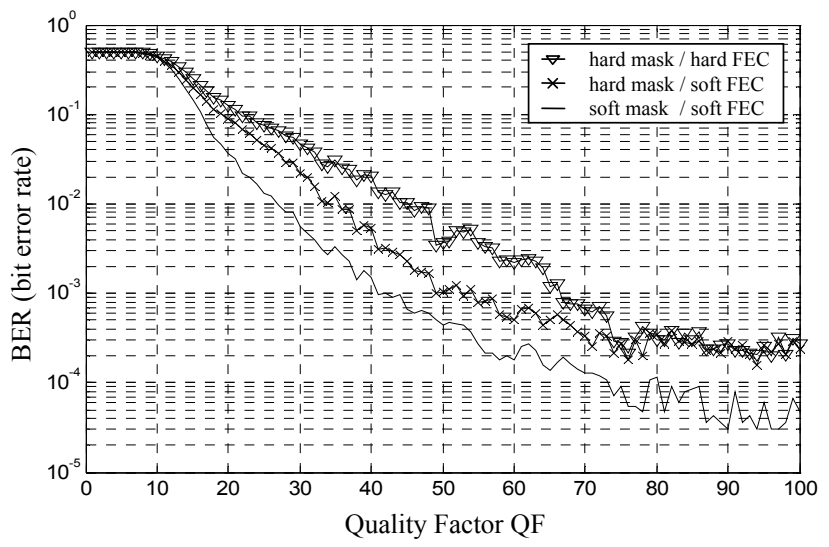

Fig. 8. BER for different values of JPEG quality factor after forward error correction. (settings as in Fig. 7).
We present results of our new solution for JPEG compressions only. Other watermark attacks, such as filtering or noise-adding, have similar effect on the probability of mask transitions and hence, functionality of our approach.

Fig. 8 shows that our soft-mask/soft-FEC solution at the decoder site outperforms watermarking using hard region separation combined with either soft or hard forward error correction. No extra coding is needed during watermark embedding. Hence, the proposed technique can be integrated very easily into numerous watermarking applications without loss of security, performance or functionality.

\section{CONCLUSION}

An integration of perceptually adaptive watermark decoding into soft-decision forward error correction was proposed. In prior watermarking approaches this adaptation process was always excluded from soft-decoding. But we proved that a soft-integration yields impressive improvements in bit error correction during watermark retrieval. No pre-distortion has to be used during watermark embedding, and hence, the image quality is not degraded. Thus, even adaptation techniques can be used where it is computational infeasible to project a pre-distortion back onto the host image.

\section{REFERENCES}

[1] R. B. Wolfgang, C. I. Podilchuk, and E.J. Delp, "Perceptual Watermarks for Images and Video," In Proc. of IEEE - Special Issue on Identification and Protection of Multimedia, vol.87 (7), pp.1108-1126, July 1999.

[2] M. L. Miller, G. J. Doërr, and I. J. Cox, "Applying Informed Coding and Embedding to Design a Robust High-Capacity Watermark," In IEEE Transactions on Image Processing, vol.13 (6), pp.792-807. June 2004.

[3] M. Schlauweg, D. Pröfrock, and E. Müller, "JPEG2000-Based Secure Image Authentication," In Proc. of ACM Multimedia and Security Workshop, Geneva, Switzerland, pp.62-67, Sept. 2006.

[4] G. Sharma, and D. J. Coumou, "Watermark Synchronization: Perspectives and a New Paradigm," In Proc. of Conf. on Information Sciences and Systems, Princeton, USA, pp.1182-1187, March 2006.

[5] J. E. Vila-Forcén, S. Voloshynovskiy, O. Koval, T. Pun, and F. Pérez-González, "Worst Case Additive Attack against Quantization-Based Data-Hiding Methods," In Proc. of SPIE Security, Steganography, and Watermark of Multimedia Contents VII, San Jose, USA, pp.136-146, Jan. 2005. 